\title{
Die neue Verfassung Ungarns
}

\author{
von Attila Vincze
}

Ungarn ist mit seinem neuen Grundgesetz in den Fokus des europäischen Verfassungsdiskurses geraten. Der Beitrag zeichnet die wichtigsten Aspekte der neuen Verfassung kritisch nach. Die Notwendigkeit einer erneuerten Verfassung, der Weg zu ihrer Verabschiedung und die bedeutendsten inhaltlichen Veränderungen werden summarisch dargestellt. Im Ergebnis steht die Erkenntnis, dass die neue Verfassung auf einer diskussionswürdigen philosophischen Grundlage geschrieben wurde und eine Reihe von Vorschriften enthält, die nur als Machtmittel der regierenden Koalition zu erklären sind. Diese negativen Merkmale dominieren das Gesamtbild, auch wenn einige Änderungen durchaus progressive Züge tragen.

Hungary attracted widespread attention within the European discourse on constitutional law in developing its Basic Law. This contribution discusses the central elements of this process, including the question of its rationale, the different steps in developing the new constitution and its key issues. In conclusion, the new constitution rests on a questionable philosophical foundation and a number of new provisions can only be conceived as instruments of political powers, especially of the ruling coalition. These negative features dominate the overall assessment of the new Basic Law, even though some progressive elements are to be discerned.

\section{Einleitung}

Ungarn ist mit seinem neuen Grundgesetz ${ }^{1}$ in den Fokus des europäischen Verfassungsdiskurses geraten. ${ }^{2}$ Dieses Interesse ist allerdings vornehmlich durch Sorgen um die Einhaltung bestimmter Werte des europäischen Verfassungsverständnisses charakterisiert. Der vorliegende Beitrag hat zum Ziel, die Kernpunkte der neuen Verfassung kritisch darzustellen. ${ }^{3}$ Bei der Auswahl der einzelnen

1 Der Ausdruck Grundgesetz und Verfassung werden im Folgenden gleichbedeutend verwendet; die Abkürzung UGG richtet sich auf das (neue) Ungarische Grundgesetz, die Abkürzung UV auf die (alte) Ungarische Verfassung.

2 Die Venedig-Kommission (Europäische Kommission für Demokratie durch Recht) legte in den Jahren 2011/2012 acht Gutachten zu aktuellen Fragen des ungarischen Verfassungsrechts vor, die überwiegend negativ ausfielen.

3 Zur umfassenden Kommentierung der alten Verfassung s. etwa: Dezsö, M./Somody, B./Vincze, A./Bodnár, E./Vissy, B.: Constitutional Law in Hungary, in Alen, A./Haljan, D. (Hg.): International Encyclopaedia for Constitutional Law, The Hague, 2010; Jakab, A.: The Republic of Hungary, Commentary, in 
Themenfelder wird versucht, die problematischsten oder auch interessantesten Neuerungen zu skizzieren und zu kommentieren.

\section{Die Notwendigkeit einer neuen Verfassung}

Die Diskussion über die neue Verfassung Ungarns wurde lange Zeit durch die kontinuierliche Verneinung der Notwendigkeit zur Novellierung bestimmt. Diesem konservativ klingenden - aber eher von Liberalen und Sozialisten vorgebrachten - Argument zufolge war eine neue Verfassung angesichts fehlenden zwingenden Bedarfs, wie er im Jahr 1989 bestand, nicht gegeben. Diesem Argument widerspricht allerdings die Tatsache, dass alle Regierungen nach der Wende versuchten, den schon aus der Präambel der alten Verfassung folgenden Auftrag zu erfüllen und eine endgültige Regelung an die Stelle der als provisorisch gedachten alten Verfassung zu setzen.

Ungarn verfügte bis 1949 über keine geschriebene Verfassung. Die mit der kommunistischen Machtübernahme erlassene und dementsprechend nicht nur den Geist, sondern auch den Text des stalinistischen sowjetischen Vorbilds von 1936 kopierende Verfassung wurde zwar während der Gespräche am Runden Tisch im Jahre 1989 grundsätzlich verändert (man könnte auch von einer Totalrevision sprechen), doch blieben zahlreiche Spuren des kommunistischen Obrigkeitsstaates im Text erhalten. Nicht nur die Struktur folgte immer noch dem sowjetischen Muster - mit einer zunehmend sinnfreien Einführung über die Grundlagen des Staates, die kaum verbundene Bereiche wie den EU-Beitritt ( $\S$ 2/A UV) und das Erbrecht ( $\$ 14$ UV) unter ein Dach stellte, gefolgt von den Bestimmungen zum Staatsorganisationsrecht und einer abschließenden stiefmütterlichen Behandlung der Grundrechte -, sondern auch der Sprachgebrauch entsprach an vielen Stellen nicht den Anforderungen an eine dem Gedanken der Gewaltenteilung verpflichtete Verfassung. Die strukturellen Eigentümlichkeiten beeinflussten die Rechtspraxis zwar kaum, ${ }^{4}$ die sprachliche Inhomogenität und die überholte Wortwahl und Stilistik ${ }^{5}$ - wie die einem hierarchisch-sozialisti-

Wolfrum, R./Grote, R. (Hg.): Constitutions of the Countries of the World, New York, 2008; vgl. auch Küpper, H.: Einführung in das ungarische Recht, München, 2011.

4 Das Urteil des ungarischen Verfassungsgerichts über die Verfassungswidrigkeit der Todesstrafe ist ein gutes Beispiel dafür; Entscheidung des Verfassungsgerichts Nr. 23/1990 (X. 31.). Die Entscheidung wurde auch auf Deutsch veröffentlicht: s. Brunner, G./Sólyom, L.: Verfassungsgerichtsbarkeit in Ungarn. Analysen und Entscheidungssammlung 1990-1993, Baden-Baden, 1995, 136-163.

5 Dazu etwa Küpper, H.: A szocialista maradványok szerepe a magyar alkotmányban, in: Fundamentum, 2007/4, 53-60; Hollán, M./Jakab, A.: Die dogmatische Hinterlassenschaft des Sozialismus im heutigen Recht: Das Beispiel Ungarn, in: Jahrbuch für Ostrecht, 1 (2005), 11-40. 
schen Staatsaufbau entsprechende Beschreibung des Parlaments als oberstem Organ der Volksvertretung - führten gleichwohl zu einer negativen Beeinflussung von Urteilen des Verfassungsgerichts. ${ }^{6}$

Zwar gab es im Jahr 2010 in Ungarn keinen Systemwechsel, doch schien es notwendig, die Unvollkommenheiten der alten Verfassung zu beheben und die "dilatorischen Formelkompromisse" der Republik oder des Verfassungsgerichts - aufzulösen und einen stilistisch konsequenten wie konzeptionell durchdachten Text zu schreiben, kurzum eine Verfassungskonsolidierung vorzunehmen. Anzumerken ist auch, dass schon die frühere sozialistische Regierung von Premierminister Gyurcsány diesen Plan verfolgte, das Vorhaben aber an der fehlenden verfassungsgebenden Mehrheit scheiterte.

Ein beachtlicher Teil der verabschiedeten neuen Verfassung - vor allem das Staatsorganisationsrecht - ist eben dieser Verfassungskonsolidierung geschuldet; an vielen Stellen beinhaltet das neue Grundgesetz kaum mehr als den Text der früheren Verfassung, ergänzt durch die zur communis opinio gewordenen Leitsätze des Verfassungsgerichts. In diesem Sinne ist die Änderung zu begrüßen. Ein weiterer Teil stellt dagegen eher das Ergebnis einer intellektuell nicht eben anspruchsvollen „Reformwut“ dar, die den Text mit überflüssiger Sakralisierung und Historisierung auffüllte, statt tatsächlich bestehende Probleme anzusprechen. Ein weiterer Teil der Änderungen ist nur durch machttechnische Überlegungen zu erklären: die Umbenennung des Obersten Gerichts zur Kurie etwa machte es etwa möglich, einen neuen obersten Richter zu wählen, wie auch die Entwicklung des Datenschutzombudsmanns hin zu einer Nationalen Behörde des Datenschutzes und der Informationsfreiheit es erlaubte, den früheren - den derzeitigen Machthabern unangenehmen - Datenschutzombudsmann abzuwählen.

\section{Grenzen der verfassungsgebenden Gewalt}

Schon im Herbst 2010, noch bevor ein Konzept für eine neue Verfassung vorlag, wurde die Frage aufgeworfen, ob die verfassungsgebende Gewalt bestimmten

\section{Entscheidung des Verfassungsgerichts Nr. 7/2001 AB (III. 14.)}

Der Ausdruck stammt von Schmitt, C.: Verfassungslehre, Berlin-Leipzig, 1928, 31f. Wie Schmitt selbst schreibt (Der Hüter der Verfassung, Berlin, 1996, 48) ,[b]ei solchen ,ddilatorischen Formelkompromissen“ ist die Entscheidung über 'Zweifel und Meinungsverschiedenheiten' in Wahrheit überhaupt erst die wirkliche Normierung." Wie es in der Tat auch in Ungarn passierte; vgl.nur die Entscheidungen des Verfassungsgerichts 48/1991 (IX. 26.), 36/1992 (VI.10.) und 47/2007 (VII.3). Die ersten zwei Entscheidungen sind auch auf Deutsch verfügbar: Brunner, G./Sólyom, L.: Verfassungsgerichtsbarkeit in Ungarn, a.a.O., 208-238, bzw. 390-411. 
Grenzen unterworfen ist. Diese theoretisch sehr interessante Frage wurde anhand eines praktischen Falles gestellt. Als das Verfassungsgericht im Herbst mit seiner Entscheidung Nr. 184/2010 (X 28.) ein rückwirkendes Steuergesetz, das bestimmte Einnahmen mit einem Steuersatz von 98 Prozent belegte, für nichtig erklärte, beantwortete die Regierungskoalition dies mit einer Verfassungsänderung, die die Kompetenzen des Verfassungsgerichts wesentlich beschränkte und es ihm verwehrte, die Verfassungsmäßigkeit von Steuergesetzen sowie der die Einnahmen und Ausgaben des Budgets beeinflussenden Gesetze zu überprüfen. Die Verfassungsmäßigkeit dieser kompetenzbeschneidenden Verfassungsänderung wurde durch mehrere Anträge in Frage gestellt.

In der Tat regelte die alte Verfassung den Prozess der Verfassungsänderung nur eher lapidar: Der einzige wirkliche Unterschied zwischen einer Verfassungsänderung und dem Erlass eines einfachen Gesetzes war die notwendige Mehrheit, die für die Verfassungsänderung bei zwei Dritteln aller Abgeordneten (§ 24. Abs. $3 \mathrm{UV}$ ), für einfache Gesetze jedoch bei der einfachen Mehrheit der anwesenden Abgeordneten lag. Diese Zwei-Drittel-Mehrheit stellt eine relativ einfache rechtliche Hürde dar; es findet sich zudem keine „Ewigkeitsklausel“, die bestimmte Themen von einer Änderung ausklammert, wie etwa Art. 79 Abs. 3 $\mathrm{GG}$, auch gibt es keine zweifache Beschlussfassung wie etwa in einem Zweikammersystem. ${ }^{8}$ Was die Zwei-Drittel-Mehrheit der Abgeordneten des ungarischen Parlaments wünscht, kann in die Verfassung aufgenommen werden. Die fehlenden inhaltlichen Grenzen der Verfassungsänderung bedeuteten auch, dass es dem Verfassungsgericht verwehrt wurde, die Richtigkeit oder "Verfassungsmäßigkeit” der Verfassungsänderung zu beurteilen. ${ }^{9}$ Dies wurde im Hinblick auf die Verfassungsänderung des Jahres 2010 bestätigt. Zwar argumentierten die Antragsteller mit der Schmitt'schen These einer Differenzierung zwischen dem pouvoir constitué und dem pouvoir constituant in dem Sinne, dass die grundsätzliche Änderung der Stellung des Verfassungsgerichts im Hinblick auf den pouvoir constitué nicht zulässig sei. Das Verfassungsgericht akzeptierte aber seine „Kastration“ ohne viel Aufsehen - eine umso erstaunlichere Haltung, als selbst in Großbritannien einige Mitglieder des House of Lords solch eine eklatante Einschränkung ihrer Kompetenzen (bzw. der des neuen Supreme Court) als un-

8 Zum Vergleich des deutschen und des ungarischen Recht siehe: Küpper, H.: Az alkotmánymódosítás alkotmánybírósági kontrollja Magyarországon és Németországban, in: Jogtudományi közlöny 2004, 265-274.

9 Vgl. die Entscheidungen des Verfassungsgerichts Nr. 1260/B/1997, 1125/I/1996. 
zulässig ansahen und dabei sogar bereit waren, die uneingeschränkte Parlamentssouveränität in Frage zu stellen. ${ }^{10}$

Die Argumentation des Verfassungsgerichts geht auf die 1989 eingeführte einfache - lediglich einen breiten politischen Konsens voraussetzende - Regel der Verfassungsänderung zurück. Während der Gespräche am Runden Tisch zwischen den kommunistischen Machthabern und der Opposition, die im Grunde genommen dazu dienten, die Grundbedingungen für freie Wahlen zu schaffen, wollte niemand eine inhaltliche Schranke für das durch kommende freie Wahlen zusammenzusetzende Parlament schaffen. Dieses dann demokratisch legitimierte Organ sollte befugt sein, eine endgültige Verfassung vorzulegen und in diesem Zusammenhang auch die Schranken für künftige Verfassungsänderungen zu setzen. Die Tatsache, dass es nach der Wende zu keiner endgültigen Verfassungsreform oder -neuschöpfung kam, ändert nichts am Sinn und Zweck dieser Regelung, auch wenn sie nach zwei Jahrzehnten einigen als unangenehm oder verfehlt erscheinen mag. ${ }^{11}$

Immerhin wird der Rahmen für Verfassungsreformen innerhalb der Europäischen Union zu einem gewissen Grad vorgegeben, sodass nicht jede beliebige Verfassungsänderung möglich ist: Solange ein Staat seine Zukunft auch als Mitglied der EU sieht, muss er die Grenzen der gemeinsamen europäischen Verfassungsüberlieferung akzeptieren.

\section{Wie wird eine Verfassung gegeben?}

Der Regierungskoalition wurde u.a. vorgeworfen, dass es keinen tatsächlichen Meinungsaustausch über die neue Verfassung zwischen ihr und der Opposition bzw. der Zivilgesellschaft gegeben hätte und die Verfassung nur die Präferenzen und Interessen der Regierungsparteien widerspiegele. Es wurde auch vorgebracht, dass die Verfassungsgebung unter solchen Umständen undemokratisch sei. Die Regierungskoalition beharrte ihrerseits auf ihrer verfassungsändernden Zwei-Drittel-Mehrheit im Parlament, die sie als per definitonem demokratisch bezeichnet.

10 Jackson v. Attorney General [2005] UKHL 56, para 102 per Lord Steyn: "In exceptional circumstances involving an attempt to abolish judicial review or the ordinary role of the courts, the Appellate Committee of the House of Lords or a new Supreme Court may have to consider whether this is a constitutional fundamental which even a sovereign Parliament acting at the behest of a complaisant House of Commons cannot abolish."

11 Näher dazu Dezsö, M./Somody, B./Vincze, A./Bodnár, E./Vissy, B.: Constitutional Law in Hungary, a.a.O., Rn. 83-87. 
Hinter dieser Auseinandersetzung steht das Dilemma der Mehrheitsdemokratie: ${ }^{12}$ Ist die Mehrheitsregel das einzige konstitutive Merkmal der Demokratie oder ist sie nur im Zusammenwirken mit einer Vielzahl von anderen Prinzipien vorstellbar? Ist die pure Mehrheitsregel auch für den Prozess der Verfassungsgebung anzuwenden oder ist hier der Konsens für die Gestaltung der Grundlagen des staatlichen Zusammenlebens unerlässlich? Ein Konsens setzt freilich eine gewisse Konsensfähigkeit, eine funktionierende Öffentlichkeit und einen Dialog zwischen den verschiedenen Seiten voraus. Die Diskurs- und Konsensunfähigkeit der ungarischen Politik - kein neuer Zustand - verhinderte in den vergangenen zwei Jahrzehnten in vielen Bereichen notwendige Reformen. Die Eigentümlichkeit der Gespräche am Runden Tisch im Jahre 1989 war, dass die damals erlassene Verfassung - teils aus der Illusion einer konsensualen Demokratie, teils aus dem gegenseitigen Misstrauen der Beteiligten an den Gesprächen - für viele Gebiete - also nicht nur zur Verfassungsänderung - eine Zwei-Drittel -Mehrheit der Parlamentsabgeordneten verlangte. So benötigten die Ausgestaltung des Streikrechts, der Versammlungsfreiheit, des Kirchenrechts, der Gerichtsverfassung, des Asylrechts oder des Kommunalrechts ebenfalls eine Supermajorität im Parlament. Diese spezielle Mehrheitsregel ist in sich mit der Idee der parlamentarischen Mehrheitsregierung kaum zu vereinbaren. Sie führte zudem dazu, dass aufgrund der Dialogunfähigkeit der parlamentarischen Parteien, entweder notwendige Änderungen und Reformen nicht verabschiedet werden konnten (etwa auf dem Gebiet des Streikrechts oder der Versammlungsfreiheit) oder die Änderungen sich auf das unbedingt notwendige Minimum beschränkten (etwa im Falle des EU-Beitritts). ${ }^{13}$

Während des jüngsten Verfassungsgebungsprozesses zeigte sich die Diskursunfähigkeit der ungarischen politischen Eliten wiederum sehr deutlich. Der regierenden Mehrheit wie auch den Oppositionsparteien war bewusst, dass das Schicksal der Verfassung vollständig vom Willen der Regierung abhängig ist, was eine wohlwollende und offene Attitüde aller Seiten notwendig gemacht hätte. Allerdings konnte keine der Parteien profitieren: Die Regierungsparteien demonstrierten ihr von einem Mangel an Respekt zeugendes Verfassungsverständnis, als sie die Verfassungsgerichtsbarkeit im Herbst 2010 wie benannt beschnitten, während die Oppositionsparteien und die mit ihnen sympathisieren-

12 Vgl. Guggenheimer, B./Offe, C.: Politik aus der Basis - Herausforderung der parlamentarischen Mehrheitsdemokratie, in: dies. (Hrsg.): An den Grenzen der Mehrheitsdemokratie, Opladen, 1984, 8-19.

13 Vgl. nun Chronowski N./Petrétei J.: EU-csatlakozás és alkotmánymódosítás: minimális konszenzus helyett politikai kompromisszum, in: Magyar jog, 2003, 449-466. 
den think tanks die Vorbereitungsarbeiten als Zeichen der Treue zur früheren Verfassung schlicht boykottierten.

So wurde nicht nur die Meinung der Opposition ignoriert, sondern auch die fachliche Öffentlichkeit aus den entscheidenden Diskussionen ausgeschlossen. Die das Grundgesetz ausarbeitende Kommission bestand aus dem Ministerpräsidenten Orbán nahestehenden Politikern, die sich eher durch ihre politische Zuverlässigkeit als durch ihre verfassungsrechtliche Expertise auszeichneten. Außerhalb dieses engen Kreises wusste man kaum etwas über die Vorbereitungsarbeiten. Zwar wurde Ende 2010 ein sehr grobes Konzept veröffentlicht, den tatsächlichen Verfassungsentwurf konnte man aber erst Mitte März 2011 lesen. Da das neue Grundgesetz bereits am 25. April 2011, am Ostermontag und am ersten Jahrestag des Erdrutschsieges der konservativen Parteien, vom Präsidenten der Republik unterschrieben wurde, gab es natürlich nicht genug Zeit, um eine vertiefte Diskussion zu führen. Die Regierungskoalition war wahrscheinlich auch nicht besonders daran interessiert, andere Meinungen zur Kenntnis zu nehmen.

Die Intransparenz der Vorbereitungsarbeiten wurde heftig kritisiert, v.a. dahingehend, dass die Geheimhaltung den Ideen einer Verfassungsgebung grundsätzlich widerspräche. Dieser Kritik ist nur in Teilen zuzustimmen: Die Philadelphia Convention von 1787, die die Verfassung der USA entwarf, war bewusst intransparent und ihre Debatten geheim, damit jeder Beteiligte offen seine Meinung bilden und vortragen konnte. Erst als der Entwurf fertig war, begann eine lebhafte Diskussion über die Vor- und Nachteile der vorgeschlagenen neuen Verfassung. Für Ungarn wird man somit sagen müssen, dass es weniger problematisch ist, dass der Entwurf der Verfassung ohne die Beteiligung der Öffentlichkeit geschrieben wurde. Als Problem ist jedoch zu benennen, dass es über diesen Entwurf keine - fachlichen oder auch nur allgemein-öffentlichen - Debatten gab. Dieser Umstand stützt die Hypothese, dass die Regierungskoalition die Verfassung eher als Mittel zur Verwirklichung ihrer eigenen Ziele ansieht und weniger als ein Produkt gesamtgesellschaftlicher Teilhabe. Dies allerdings führte dazu, dass die Verfassung ihre integrative Funktion kaum wird erfüllen können.

\section{Eine neue Staatsauffassung und die Präambel der Verfassung}

Die in der neuen Verfassung zutage tretende Staatsauffassung lässt sich in drei Schlagworten zusammenfassen: staatliche Zentralisierung (1), nationalisierende Idealisierung (2) und Sakralisierung (3). 


\section{Zentralisierung}

Schon vor den Wahlen im Jahre 2010 war von Politikern und Intellektuellen des rechten Spektrums zu hören, dass der ungarische Staat nach den Wahlen völlig neu organisiert werden müsse. Die weit verbreitete Korruption und der Klientelismus sowie die nur bedingt professionell agierende staatliche Verwaltung etwa im Polizeiwesen oder auch bei den Sozialleistungen - wurden der liberalen Ausrichtung früherer Regierungen zugeschrieben. New Public Management, contracting-out, schlanker Staat, PPP-Techniken und marktkonforme Leistungsverwaltung wurden als etwas grundsätzlich Schädliches eingestuft, ein neues Staatsbild verlangt. ${ }^{14}$

Das Schlagwort der neuen Staatsauffassung lautete „Neo-Weberianismus“ verbunden mit der Grundposition, die dem Staat wieder eine zentrale Rolle zusprach. Er sollte zudem eine wichtige ökonomische Funktion erfüllen und sich nicht mehr damit begnügen, die Wirtschaft lediglich als regulator oder facilitator zu beeinflussen. Stattdessen sprach man sich für ein aktives wirtschaftliches Engagement aus - ein Gedanke, der mitten in der Wirtschaftskrise für viele recht sympathisch klang. Die avisierte Einschränkung der Wirkungen des freien Marktes lässt sich an zahlreichen Maßnahmen der neuen Regierung dokumentieren, etwa an der Verstaatlichung der privaten Pensionsfonds, am Ankauf eines großen Aktienpakets der ungarischen Ölgesellschaft $\mathrm{MOL}^{15}$ oder am Auftreten eines staatlichen Anbieters in der Telekommunikation.

Der Zentralisierungsschub war auch an der Spitze der Regierung zu spüren: Die Zahl der Ministerien wurde nahezu halbiert, wichtige Ressorts - wie Gesundheit oder Kultur - sind nur noch durch Staatssekretäre vertreten. Das Kabinett funktioniert weniger als Kollektiv denn als „Generalstab“, der die Befehle der Leitung befolgt, so im Rahmen der Entscheidung über die Zahl der staatlich finanzierten Ausbildungsplätze für Juristen, deren Verringerung vom Ministerpräsidenten persönlich verfügt wurde.

Die weitgehende Zentralisierung bedeutet auch eine Verteilung von Führungspositionen nach parteipolitischer Loyalität, ein langfristiges Charakteristikum ungarischer Staatlichkeit, unabhängig von der politischen Färbung. Im Jahr 2004,

14 Zum Thema Staatsbild vgl. Voßkuhle, A.: Der ,Dienstleistungsstaat': Über Nutzen und Gefahren von Staatsbildern, in: Der Staat, 40 (2001), 495-523.

15 Dieser Gesellschaft wurden schon früher Privilegien gewährt, z.B. durch die sogenannte Lex Mol, die eine feindliche Übernahme praktisch unmöglich machte siehe dazu: Vincze, A.: A "lex MOL" és a töke szabad mozgása az Európai Bíróság gyakorlata tükrében, in: Gazdaság és jog 2008/2, 3-9. 
kurz nach der Amtseinführung von Gyurcsány, wurde das Gesetz CXXVI/2004 erlassen, um die Zahl der Mitglieder des Monetären Rates, des wichtigsten Entscheidungsorgans der Zentralbank, zu erhöhen und eine linksorientierte Dominanz gegen den damaligen rechtsorientierten Präsidenten der Zentralbank, Járai, zu ermöglichen. ${ }^{16}$ Ein ähnliches Ziel verfolgte das Gesetz CCVIII/2011, das die Wahl der Mitglieder des Monetären Rates dem - derzeit von der Regierung dominierten - Parlament überträgt. Die parteipolitische Schattierung der ,unabhängigen“ Institutionen beschränkt sich aber nicht auf die Zentralbank, sondern erstreckt sich auch auf den - inzwischen von einer Plagiatsaffäre betroffenen Präsidenten der Republik, die Präsidentin des nationalen Medienrates und einige neue Verfassungsrichter. Auch der Präsident des Rechnungshofes ist ein führender Politiker der Regierungspartei. Zudem ist die Neugestaltung der Gerichtsverwaltung mit den herkömmlichen Auffassungen von Rechtsstaatlichkeit nicht einfach zu vereinbaren. Die durch das Gesetz CLXI/2011 geschaffene neue Verwaltung wird durch den Präsidenten der Gerichtlichen Landesbehörde dominiert, der vom Parlament für neun Jahre gewählt wird und in den meisten gerichtsspezifischen Angelegenheiten allein entscheidet. Diese Behörde, mit grundsätzlichen Entscheidungsbefugnissen ausgestattet, wird in der Verfassung nirgends erwähnt, ihr Existenzrecht wird auch dadurch in Frage gestellt, dass die Präsidentin dieser Behörde die Frau eines einflussreichen Politikers der Regierungspartei ist, der selbst an der Ausarbeitung der Verfassung beteiligt war. ${ }^{17}$

\section{Nationale Ideologisierung}

Die neue Staatsauffassung lebt ganz vom Gedanken der nationalen gloire. Die Präambel der Verfassung wurde in „Nationales Glaubensbekenntnis“ umbenannt, ein Ausdruck, der recht einfach mit der herrschenden revisionistischen ungarischen Ideologie zwischen den zwei Weltkriegen in Verbindung gebracht werden kann $^{18}$ - unbeschadet des tatsächlichen Willens der Autoren der Verfassung -

16 Zur deutschsprachigen Diskussion: Häde, U.: Unabhängigkeit für die ungarische Nationalbank?, Zum Status der Zentralbanken von Mitgliedstaaten mit Ausnahmeregelung, in: Europäische Zeitschrift für Wirtschaftsrecht, 16 (2005), 679-682.

17 Vgl. auch das Gutachten der Venedig-Kommission: CDL-AD(2012)001 Opinion on Act CLXII of 2011 on the Legal Status and Remuneration of Judges and Act CLXI of 2011 on the Organisation and Administration of Courts of Hungary, adopted by the Venice Commission at its 90th Plenary Session (Venice, 16-17 March 2012).

18 Der Ausdruck „Nationales Glaubensbekenntnis“ evoziert eine nach dem Ersten Weltkrieg geschriebene ungarische Paraphrase des Apostolischen Glaubensbekenntnisses, welches - in deutscher Übersetzung - 
und zudem den Eindruck erweckt, dass man sich mit diesem langen, pathetischen und oft kitschigen Text unbedingt identifizieren solle, wenn man zur Nation gehören möchte. Das „Bekenntnis“ suggeriert aber eine Staats- und Geschichtsauffassung, die nicht für alle akzeptabel ist. Auch deshalb scheitert die Verfassung als gemeinsamer Nenner aller Bürgerinnen und Bürger.

Die Präambel unterstreicht mehrmals den christlichen Glauben und die Nation als die wichtigsten Bezugspunkte. Die Überbetonung dieser Werte, möglicherweise als Kompensation fehlender Innovationen in der den Neubeginn symbolisierenden Verfassung verstanden, entspricht zwar nicht dem Zeitgeist postnationaler Konstellationen ${ }^{19}$ und mag auf einige Staatsbürger irritierend wirken, ist jedoch nicht beispiellos in Europa. Viele Verfassungen beinhalten Bezüge zur wahren oder auch nur mythologisierten Geschichte ${ }^{20}$ oder zu einem bestimmten Glauben. ${ }^{21}$ Deshalb ist die Erwähnung der heiligen Krone, oder die Rolle des Staatsgründers, des heiligen Stephans, in der Präambel weniger problematisch. Die in dem Nationalen Bekenntnis hervorgehobene historische Rolle des Christentums in der ungarischen Geschichte ist - als Tatsache - auch nicht zu bestreiten: Ohne die Übernahme des in Westeuropa damals herrschenden Glaubens am Ende des 10. Jahrhunderts hätte Ungarn kaum lange existiert, sondern wäre von den deutschen Rittern erobert worden. ${ }^{22}$

Die Aberkennung der Legitimität und der Legalität des kommunistischen Regimes im „Nationalen Glaubensbekenntnis“ und die Annahme, dass die ungarische Staatlichkeit seit der deutschen Machtübernahme am 19. März 1944 suspendiert war, sind ebenfalls keine Feststellungen, die man unbedingt ablehnen müsste, auch wenn diese Geschichtsauffassung den Versuch der Demokratisierung in Ungarn zwischen 1945-1948 komplett ausblendet und nicht ganz der Wirklichkeit entspricht. Aus rein juristischer Sicht ist es eher problematisch, die Frage zu beantworten, was diese Suspendierung nun bedeutet, welche Konsequenzen sie hat. Ist somit alles, was zwischen dem 19. März 1944 und dem 2.

lautet, wie folgt: „Ich glaube an Gott, Ich glaube an das Vaterland, Ich glaube an die Auferstehung Ungarns“ („Hiszek egy Istenben, hiszek egy Hazában, Hiszek Magyarország feltámadásában“).

19 Vgl. nur Habermas, J.: Die postnationale Konstellation, Frankfurt/Main, 1998.

20 Die kroatische Verfassung beginnt etwa mit einer langen Erzählung der Geschichte von Kroatien, die Präambel der slowakischen Verfassung enthält Bezüge zum Großmährischen Reich als Vorgänger der Slowakischen Republik - beides eher Ausnahmen als die Regel.

21 Die irische Verfassung leitet alle Macht von Gott ab, die griechische wurde im Namen der Dreifaltigkeit erlassen, die Verfassung von Malta bestimmt den römisch-apostolischen Glauben als den Glauben von Malta, die polnische Verfassung und sogar das deutsche Grundgesetz enthalten einen Gottesbezug.

22 Was 1051 von Kaiser Heinrich III. immerhin versucht wurde. 
Mai 1990 geschah, nur illegitim oder auch illegal? Falls nur illegitim, dann sagt die neue Verfassung wiederum nicht mehr als die Entscheidung des Verfassungsgerichts Nr. 11/1992 (III.5), soweit aber die Suspendierung Illegalität bedeutet, wäre eine Reihe weiterer Fragen zu beantworten.

Das Einführungsgesetz zum ungarischen Grundgesetz enthält ausführliche Bestimmungen über die Verantwortlichkeit der früheren Staatspartei für das Unrecht des kommunistischen Obrigkeitsstaates, erklärt aber keine Rechtsvorschrift für ungültig, die nach dem 2. März 1944 entstanden ist. Das Einführungsgesetz erklärt jedoch die in einem Ausführungsgesetz zu bestimmenden Straftaten als nicht verjährt. In dieser Hinsicht bringt das Einführungsgesetz etwas Neues: Immerhin ist zu erwarten, dass das zu erarbeitende Ausführungsgesetz eher den politischen Interessen der Regierungspartei dienen wird als den Anforderungen der Gerechtigkeit.

Die Betonung von Werten wie Gerechtigkeit, Billigkeit, Freiheit, nationale Schicksalsgemeinschaft, Glaube, Treue, Liebe, Würde usw. in der Präambel klingt zwar pathetisch und an einigen Stellen sogar kitschig, doch stellte dies kein besonderes Problem dar, wenn das Grundgesetz nicht im Einklang mit diesem Nationalen Glaubensbekenntnis und mit der historischen Verfassung auszulegen wäre. Der Verfassungstext selbst ist nämlich überfrachtet mit Wertentscheidungen, sehr offen formuliert, und oft sind es besonders knapp dargelegte Bestimmungen, die eine Reihe von Interpretationsproblemen bergen. Diese Probleme potenzieren sich, da die entsprechenden Vorschriften im Lichte des noch offener und einen breiteren Wertungsspielraum einräumenden „Bekenntnisses“ zu interpretieren sind. Die Auslegung im Lichte der Präambel ist nicht nur methodisch fraglich, sondern hat auch einen faden Beigeschmack: Die in Ungarn lange Zeit herrschende kommunistische Partei verlangte auch, Rechtsvorschriften ideologisch auszulegen - ein Merkmal, das der Kommunismus mit dem Nationalsozialismus teilte. ${ }^{23}$ Die moralische Erneuerung, die sich das ungarische Grundgesetz zum Ziel gesetzt hat, ist aber nur durch strikte Rechtsbindung der Staatsgewalt möglich und nicht durch eine Auflösung der Kompetenzgrenzen in ideologischen Zauberformeln.

Die historische Verfassung, die bis 1944 die Grundlage des ungarischen Verfassungsverständnisses bildete, stellte leider auch keine bessere Auslegungsmaxime dar, hatte sie doch viel mit dem britischen Verfassungsverständnis gemein: Es

23 Vgl. nur Rüthers, B.: Die unbegrenzte Auslegung, Tübingen, 2005. 
gab keinen einheitlichen Verfassungstext, der eine herausragende, die anderen Gesetzen überragende Position gehabt hätte, sondern eine Reihe von wichtigen Gesetzen, die aus historischen und politischen Gründen eine besondere Rolle spielten. Sie galten als Teile der Verfassung: die goldene Bulle von 1222, die Pragmatica Sanctio oder auch die Reformgesetze der Revolution 1848. Diese Gesetze wurden durch bestimmte Doktrinen - wie die Doktrin der Heiligen Krone - und das Gewohnheitsrecht ergänzt. Die historische Verfassung war also organisch, hat sich in der praktischen Anwendung bewiesen und überlebt. Ein nationaler Romantizismus kann zwar von einer Rückkehr zu diesem Konzept sprechen, faktisch ist dieser Schritt jedoch nicht durchzuführen, da eben jene alltägliche Praxis, die die vitale Kraft der alten Verfassung darstellte, seit fast sieben Jahrzehnten fehlt. Jegliche Bezugnahme auf dieses historische Recht wird deshalb selektiv und subjektiv sein, ganz nach Bedarf und Situation. Das ungarische Grundgesetz geht aber leider noch weiter und verlangt von den (Verfassungs-)Richterinnen und Richtern, dass sie die Gesetze im Lichte der Errungenschaften der historischen Verfassung auslegen. Was eine Errungenschaft ist, bleibt dem Richter vorbehalten. Zudem hätte es eines entsprechenden Textes nicht bedurft, da das Verfassungsgericht in den wenigen Fällen, in denen die historische Verfassung eine Hilfe war, bereits eine entsprechende historische Auslegung vorgenommen hatte. ${ }^{24}$

\section{Sakralisierung}

Die Sakralisierung der Verfassung steht zwar in der Nähe der Ideologisierung, doch erscheint es notwendig, das sich damit verbindende Problem vertieft darzustellen. Was auffällt, ist der oft wiederholte Gottes- und Schicksalsbezug in der Verfassung:

- Das ungarische Grundgesetz beginnt mit einer Zeile der 1823 geschriebenen ungarischen Hymne „Gott, segne den Ungar!“

- Es bezieht sich zudem auf eine Verfassungsschöpfung im Bewusstsein vor Gott und den Menschen, was die Präambel des deutschen Grundgesetzes paraphrasiert. Dadurch wird der Eindruck zumindest nicht vermieden, dass die Lage im besiegten Nachkriegsdeutschland mit der Ungarns nach den sozialistischen Regierungen in Zusammenhang gebracht werden soll.

24 Vgl. nur die Entscheidungen des Verfassungsgerichts 48/1991. (IX. 26.), 2/1994. (I. 14.) und 47/2009. (IV. 21.). Die deutsche Übersetzung der ersten Entscheidung ist erschienen in: Brunner, G./Sólyom, L., a.a.O., 208-238. 
- Die Präambel betont die Notwendigkeit der moralischen Erneuerung Ungarns und impliziert, dass das neue Grundgesetz, das nicht gerade frei von parteipolitischen Überlegungen ist, diese Erneuerung bringen soll.

- Die Präambel fasst das Grundgesetz ausdrücklich als ein Vertrag zwischen der Vergangenheit, der Gegenwart und der Zukunft auf.

- $\quad$ Nicht nur der Text ist sakralisiert, sondern auch der um die neue Verfassung konstruierte Kult. Es wurde „ein Tisch des Grundgesetzes“ in jeder Stadt und jeder Gemeinde aufgestellt, an dem die Bürger das Grundgesetz lesen können. Die Regierung bestellte zudem eine Reihe von Gemälden, die bestimmte Epochen der ungarischen Geschichte darstellen und eine Festausgabe des Grundgesetzes schmücken sollen.

Eine Verfassung ist kein Allheilmittel für gesellschaftliche Probleme, auch wenn die Regierung sie so interpretiert. Die überbetonte Ideologisierung der Verfassung hilft sicher nicht, eine wie auch immer definierte materielle Integration $\mathrm{zu}$ verwirklichen, sondern lässt eher Milan Kunderas Worte von der ,unerträglichen Leichtigkeit des Seins“ Wirklichkeit werden. „Wo aber eine einzige politische Partei alle Macht hat, befinden wir uns im Reich des totalitären Kitsches.“

\section{Grundrechte}

Kritisch vorgebracht wurde schließlich, dass das neue ungarische Grundgesetz ein dem europäischen Verständnis fremdes Grundrechtsverständnis aufweist. Dieser scharfen Kritik muss nachgegangen werden, auch weil eine Reihe der Grundfreiheiten nach dem Vorbild des Textes der Grundrechte-Charta der EU verfasst wurden (Art. III UGG).

Schon die Präambel betont, dass sich die individuelle Freiheit nur im Zusammenwirken mit anderen entfalten kann und dass die wichtigsten Rahmen des Zusammenlebens die Familie und die Nation sind. Schon diese Zeilen zeugen von einer Abwehr des Individualismus als philosophischer Grundlage der Grundrechte. Diese veränderte Auffassung ist in einigen Vorschriften deutlich zu spüren, die - den Forderungen von rechtsorientierten Publizisten und Intellektuellen folgend - eher die Pflichten als die Rechte betonen: nach Art. XII Abs. 1 UGG ist jeder verpflichtet, durch eine den Möglichkeiten und der Begabung entsprechende Arbeit zur Bereicherung der Gemeinschaft beizutragen. Nach Art. XVII Abs. 1 UGG müssen die Arbeitgeber und die Arbeitnehmer im Interesse der Arbeitsplatzsicherung, der Nachhaltigkeit der Volkswirtschaft und anderer gemeinschaftlicher Ziele zusammenarbeiten, was dem in Art XVII Abs 2 UGG garantierten Streikrecht einen ganz neuen - eher korporatistischen - Inhalt verleiht. Was bedeutet Streikrecht, wenn es einer Pflicht unterliegt, die Nachhaltig- 
keit der Volkswirtschaft zu berücksichtigen? Eine weitere bedingungslose Verfassungspflicht besteht für Kinder, denen verpflichtend auferlegt wird, für ihre notleidenden Eltern zu sorgen (Art. XVI. Abs. 4 UGG), unabhängig davon, wie sich die Eltern den Kindern gegenüber verhalten haben. So ist also auch ein Alkoholiker, der das Leben seiner Kinder durch physischen Terror und gegebenenfalls auch durch Missbrauch zur Hölle machte, fortan berechtigt, von diesen Unterstützung zu verlangen und notfalls einzuklagen. Diese verfassungsrechtlich verankerten Pflichten gehen weit über das hinaus, was aus einer Sozialbindung des Eigentums gesamteuropäisch angenommen wird. ${ }^{25}$

Die Betonung der Pflichten gegenüber der Gesellschaft bedeutet aber nicht, dass keine individuellen Freiheitsrechte in den Grundrechtekatalog aufgenommen wurden. Das Grundgesetz enthält weiterhin die Versammlungs- und Vereinigungsfreiheit (Art. VIII UGG), das Recht auf freie Meinungsäußerung (Art. IX UGG) und auf Privatsphäre (Art. VI UGG). Das Problem mit diesen Rechten, besser gesagt mit ihrer Verschriftlichung, bleibt im Vergleich zur vorhergehenden Verfassung unverändert: Das Grundgesetz statuiert zwar immer einen Grundsatz, ein Recht auf etwas oder eine Freiheit von etwas, sagt aber - abgesehen von einer generellen Regel der Grundrechtsbeschränkung - nichts oder kaum etwas über die Schranken der einzelnen Rechte und Freiheiten. Die Ausgestaltung dieser Grundrechte bleibt dem Parlament und dem Verfassungsgericht vorbehalten.

Dies schließt jedoch nicht aus, dass sich eine den europäischen Standards entsprechende Bedeutung der Grundrechte in der Praxis entfalten könnte. Der Verfassungstext gibt dafür genug Anhaltspunkte: Art. Q etwa verlangt, dass das innerstaatliche ungarische Recht mit den völkerrechtlichen Verpflichtungen übereinstimmen muss, woraus eine völkerrechtskonforme Auslegung des ungarischen Rechts folgt. Die Einbeziehung dieser Standards wäre sicher notwendig bei der Auslegung bestimmter Vorschriften, wie im Falle von Art. IV Abs. 2 S. 2 UGG, nach dem eine tatsächliche lebenslange Freiheitsstrafe ${ }^{26}$ nur aufgrund vorsätzlich und gewaltsam begangener Straftaten zu verhängen ist. Tatsächliche lebenslange Freiheitsstrafen sind zwar in Europa nicht unbekannt, bilden aber eher eine Ausnahme. Art 123a. der Schweizerischen Verfassung kennt diese

25 Vgl. GG Art. 14, Art. XIII. Abs. (1) UGG; aus der Praxis des EuGH vgl. nur die klassischen Fälle EuGH Rs. 44/79, Slg. 1979, 3727 - Hauer; EuGH Rs. 4/73, Slg. 1974, 491.

26 Tatsächliche lebenslange Freiheitsstrafe ist ein Freiheitsentzug, der aus keinem Grund zu unterbrechen oder frühzeitig zu beenden ist. 
Strafe für Sexual- oder Gewaltstraftäter, die als extrem gefährlich erachtet und als nicht therapierbar eingestuft werden. Eine vergleichende Studie, die auf dem EGMR-Urteil vom 17. Januar 2012 Vinter and Others v. The United Kingdom (Rn. 51-73) aufbaut, zeugt davon, dass unbefristete Freiheitsstrafen nur ausnahmsweise mit der EMRK zu vereinbaren sind. Die sehr breit gefassten Formulierungen in der ungarischen Verfassung - ,vorsätzlich und gewaltsam begangen“ - erfüllen diese Bedingungen sicher nicht. Das ungarische Grundgesetz sagt aber nicht, dass alle vorsätzlich und gewaltsam begangenen Straftaten mit einem lebenslangen Freiheitsentzug zu bestrafen wären. In diesem Fall hat der parlamentarische Gesetzgeber große Spielräume, die Bedingungen der lebenslangen Freiheitstrafe auszugestalten, auch wenn er laut Art. Q UGG den völkerrechtlichen Verpflichtungen nachkommen muss.

In dieser Hinsicht gibt es begründete Zweifel. Als das Kirchenrecht im Jahr 2011 durch das Gesetz C/2011 neugestaltet wurde, ${ }^{27}$ kam es in zweierlei Hinsicht zu Änderungen, die mit herkömmlichen Standards schwer zu vereinbaren sind. Einerseits entschied das Parlament über die Registrierung religiöser Gruppierungen als Kirchen, was aus einem Recht schlicht eine Gnade macht. Andererseits wurden diejenigen Kirchen, die keine parlamentarische Registrierung benötigen, in einem Anhang des Gesetzes bestimmt. Diese Liste umfasst aber nur die etablierten jüdischen und christlichen Glaubensgemeinschaften und schloss damit muslimische, buddhistische oder hinduistische Gruppen ohne Rücksicht auf ihre tatsächliche soziale Verankerung aus. Eine derartige Auslese ist mit der Idee des säkularen Staates und der Religionsfreiheit kaum zu vereinbaren.

Interessanterweise wurden die klassisch-konservativen „Reizthemen“, etwa die gleichgeschlechtliche Ehe oder das Recht auf Leben (bzw. das Abtreibungsverbot), nicht neu formuliert. Nach Art. L UGG ist die Ehe zwar nur zwischen einem Mann und einer Frau möglich, doch birgt diese Vorschrift nichts Neues, da sich das Verfassungsgericht im Jahr 2008 inhaltsgleich äußerte. ${ }^{28}$ Einige europäi-

27 Das Gesetz wurde inzwischen vom Parlament - kurz vor seiner Nichtigerklärung durch das Verfassungsgericht - selbst zurückgezogen und durch ein neues Gesetz ersetzt, das Gesetz Nr. CCCVI aus dem Jahr 2011. Vgl. hierzu: Opinion On Act CCCVI of 2011 on the Right to Freedom of Conscience and Religion and the Legal Status of Churches, Denominations and Religious Communities of Hungary adopted by the Venice Commission at its 90th Plenary Session (Venice, 16-17 March 2012) Opinion 664/2012 CDL-AD (2012)004. Die parlamentarische Rücknahme des Gesetzes ist dem Umstand zuzuschreiben, dass einige der neu gewählten Verfassungsrichter, gleichsam als Zeichen einer durch solche Kleinigkeiten wie die Wahl zum Verfassungsrichter nicht erschütternden Treue, die Regierung über das zu erwartende Urteil informierten.

28 Entscheidung des Verfassungsgerichts Nr. 154/2008 (XII. 17.) 
sche Verfassungen sehen gleichlautende Regeln vor, wie etwa Art. 18 der polnischen oder Art. 38 der litauischen Verfassung. Diese Vorschriften sind mit der EMRK vereinbar, da Art. 12 EMRK das Recht der Eheschließung für Männer und Frauen gemäß den einschlägigen nationalen Gesetzen einräumt (siehe Schalk/Kopf v. Austria, Application No. 30141/04). ${ }^{29}$

Auch der dem Leben des Fötus ab dem Zeitpunkt der Empfängnis zukommende Schutz ist nicht so weit gefasst wie im irischen Verbot der Schwangerschaftsunterbrechung formuliert (Art. 40 Abs. 3 Irische Verfassung) und geht keineswegs über das hinaus, was aus der bisherigen Rechtsprechung des Verfassungsgerichts folgt. $^{30}$

Die Sozialrechte hingegen erfuhren eine Änderung. Die frühere Verfassung folgte noch dem sowjetischen Sprachgebrauch der sozialen Rechte und einer Reihe von Wohlfahrtsleistungen wurde Grundrechtsstatus gewährt: die Rechte auf psychische und physische Gesundheit, auf Arbeit, auf Kultur wurden als klassische Rechte aufgefasst, die auch die Höherwertigkeit der sozialistischen Gesellschaft untermauern sollten. Der Staat war nach der Wende jedoch finanziell nicht in der Lage, die Mehrzahl der Wohlfahrtsdienstleistungen verfassungsgemäß zu gewährleisten. Als die Verwirklichung der sozialen Rechte gerügt wurde, deutete das Verfassungsgericht diese als Staatszielbestimmungen um und beschränkte ihren Inhalt auf ein Minimum. Das neue Grundgesetz leistet nichts anderes als die Verschriftlichung dieser Praxis, der zufolge sich der Staat um ein hohes Niveau der Wohlfahrtsdienstleistungen bemüht. Es gibt kein Recht auf Arbeit mehr, nur die Vorgabe, die Bedingungen für Vollbeschäftigung zu erreichen (Art. XII UGG), zudem kein Recht auf soziale Sicherheit, nur das Ziel, die soziale Sicherheit zu gewährleisten (Art. XIX UGG). Die Verfassungsväter hätten statt all dieser langen und inhaltslosen Bestimmungen ein einziges Substantiv in die Verfassung aufnehmen können: Ungarn ist ein Sozialstaat.

\section{Verfassungsgerichtsbarkeit}

Das neue Grundgesetz hat auch die Kompetenzen des Verfassungsgerichts in zwei Richtungen neu zugeschnitten. Einerseits wurde die Verfassungsbeschwerde nach deutschem Muster eingeführt - als „echte Verfassungsbeschwerde“, wie

29 Mit gleichem Erogenous: Commission for Democracy Through Law (Venice Commission) Opinion on the New Constitution of Hungary, Adopted by the Venice Commission at its 87th Plenary Session (Venice, 17-18 June 2011) Nr. CDL-AD (2011) 016, Rn. 46-50.

30 Entscheidung des Verfassungsgericht Nr. 64/1991. (XII. 17.), Brunner, G./Sólyom, L., a.a.O., 256-305. 
sie in der ungarischen Literatur bekannt ist. Anderseits wurde die actio popularis abgeschafft. Die erste - schon seit langem verlangte - Änderung wurde positiv aufgenommen, die zweite eher kritisiert. Eine dritte wesentliche Änderung betraf die Zusammensetzung des Verfassungsgerichts; die Zahl der Verfassungsrichter wurde um vier auf fünfzehn erhöht, was - nicht völlig unbegründet - als eine Machtübernahme durch politisch zuverlässige Richter interpretiert wird.

Eine Verfassungsbeschwerde war in Ungarn früher nur aufgrund der Verfassungswidrigkeit eines im konkreten Verfahren angewandten Gesetzes möglich. Die verfassungswidrige Anwendung eines Gesetzes etwa durch ein Gerichtsurteil oder einen Verwaltungsakt war - im Gegenteil etwa zur deutschen Praxis ${ }^{31}$ kein statthafter Gegenstand einer Beschwerde. Dies wurde als eine grundsätzliche Lücke im verfassungsgerichtlichen Rechtschutz angesehen. Die deutsche Praxis zeigt immerhin, dass Verfassungsbeschwerden nur dann eine realistische Chance auf Erfolg haben, wenn die Beschwerde die Verfassungswidrigkeit einer angewandten Rechtsvorschrift anmahnt. Somit war die tatsächliche Lücke in Ungarn nicht so breit wie allgemein angenommen.

Ein weiteres Merkmal des früheren Modells der Verfassungsgerichtsbarkeit war die Dominanz der actio-popularis-Verfahren. Die frühere Verfassung und ihre großzügige Auslegung ${ }^{32}$ sicherte allen das Recht - ohne jegliches rechtliches Interesse - ein nachträgliches abstraktes Verfahren zur Nichtigerklärung einer Norm einzuleiten. Dies wurde auf der einen Seite als Beweis für die weiten Kompetenzen des ungarischen Verfassungsgerichts angesehen. Hans Kelsen selbst meinte, dass die stärkste Garantie der Verfassungsgerichtsbarkeit die actio popularis sei, auch wenn er selbst diese Lösung wegen der möglichen Überlastung des Verfassungsgerichts nicht empfahl. ${ }^{33}$ Die - unvermeidbare - Überflutung mit Anträgen hatte aber auch eine Reihe von weiteren negativen Folgen: die Überpolitisierung des Gerichts und eine immer weiter sinkende Qualität der Entscheidungsbegründung. Die Politisierung betraf nicht nur die Auswahl der letztendlich entschiedenen Fälle, ${ }^{34}$ sondern auch die Rechtsfolgen der Verfassungswidrigkeit in dem Sinne, dass das Gericht trotz einer festgestellten Verfas-

31 Siehe etwa Hillgruber, C./Goos, C.: Verfassungsprozessrecht, Heidelberg, 2006, Rn. 154-160.

32 Entscheidung des Verfassungsgerichts 4/1997. (I. 22).

33 Vgl. Kelsen, H.: Wesen und Entwicklung der Staatsgerichtsbarkeit, in Veröffentlichungen der Vereinigung der Deutschen Staatsrechtslehrer, 1929, 74. Aus der ungarischen Literatur vgl. etwa Lábady, T.: A populáris akció és az egyéni jogvédelem biztosítása az alkotmánybírósági eljárásban, Magyar Jog 1991, 388; ders.: A magyar Alkotmánybíróságról szóló törvény, Jogtudományi Közlöny 1992, 288.

34 Die Todesstrafe wurde praktisch sofort durch die Entscheidung 23/1990 (X. 31.) abgeschafft, auf die Euthanasie-Entscheidung - 22/2003 (IV. 28.) - musste man zehn Jahre warten. 
sungswidrigkeit einer Norm auf die Nichtigerklärung verzichtete und sich frivoler Weise mit der Feststellung der Versäumnis einer verfassungsmäßigen Regelung begnügte. ${ }^{35}$ Die hohe Zahl von Anträgen und die Notwendigkeit, die Fälle so schnell als möglich abzuschließen, führte - dank der elektronischen Textverarbeitung - zu einer Ausschneiden-Kopieren-Einfügen-Argumentation. Die actio popularis-Verfahren wirkten auf das Verfassungsgericht gleichsam pathologisch und dienten oft nur der Durchsetzung derjenigen politischen Interessen, die im normalen parlamentarischen Prozess kaum eine Chance hatten. ${ }^{36}$ Im Hinblick auf all diese negativen Tendenzen ist es eher zu begrüßen, dass actio popularis-Verfahren abgeschafft wurden.

\section{VIII.Finanzverfassung und die Begrenzung der Staatsverschuldung}

Die frühere sozialistische Regierung verlor im Herbst 2006 nicht nur aufgrund der massenhaften und systematischen Grundrechtsverletzungen gegen die damaligen Demonstranten die Macht, sondern auch als Ergebnis ihrer finanzpolitischen Maßlosigkeit und des seit 2002 angehäuften Schuldenberges, der nicht mehr zu beherrschen war und im Jahr 2008 zum wirtschaftlichen Zusammenbruch des Landes führte. Eine neue Finanzverfassung war notwendig, die den Ratschlägen von Polonius folgt: „Kein Borger sei und auch Verleiher nicht; Sich und den Freund verliert das Darlehn oft, und Borgen stumpft der Wirtschaft Spitze ab.“

Die frühere Finanzverfassung bestand aus einigen sporadischen Anmerkungen in verschiedenen Kapiteln der alten Verfassung, die nicht zu einem systematisch durchdachten Kapitel zusammengefasst waren. Dementsprechend hatten die Vorschriften auch keine besonders große Direktionswirkung. Im Fahrwasser der Finanzkrise wurde zwar ein Gesetz zur Unterstützung der Wirtschaftlichkeit des Haushalts erlassen, das aber kaum praktische Relevanz erfuhr. ${ }^{37}$

Die neue Regierung versprach auch in diesem Bereich Tatkraft und Reformen. Das im Jahre 2011 erlassene Grundgesetz enthält zwar ein Kapitel mit dem Titel

35 Etwa die Entscheidung des Verfassungsgerichts 33/2006 (VII. 13.). Aus der Literatur Vincze, A.: 32/A Az Alkotmánybíróság, in: Jakab, A. (Hg.): Az Alkotmány kommentárja, Budapest, 2009, Rn. 155.

36 Klassisch formuliert: Griffith, J.A.G.: The Political Constitution, in: Modern Law Review 42 (1979), 17: "Instead there are political claims by individuals and by groups. One danger of arguing from rights is that the real issues can be evaded. What are truly questions of politics and economics are presented as questions of law.[...] It seems to me that to call political claims "inherent rights" is to mythologise and confuse the matter."

37 Dazu Vincze, A.: A takarékos állami gazdálkodásról szóló törvény - sok hűhó semmiért?, in: Közjogi Szemle 2009/1. 31-38 
„Öffentliche Finanzen“, der Inhalt dieser Vorschriften ist aber nur aus einem Aspekt neu: Es wird eine Verschuldungsgrenze eingeführt. Nach dieser Verschuldungsgrenze darf das Parlament kein Budget verabschieden, das zu einer Verschuldung höher als die Hälfte des Bruttosozialproduktes führt (Art. 36 Abs. 4 UGG). Diese Verschuldungsgrenze ist zwar wesentlich niedriger als die der EU (in Art. 126 AEUV verankert), im Gegensatz zur europäischen bezieht sich die ungarische Regel aber nicht auf die Verschuldung der Kommunen, die einem anderen Regime unterliegen (Art. 34 Abs. 5 UGG). Zudem verliert sie auch kein Wort über die Höhe des Haushaltsdefizits. Zur Garantie der Wirksamkeit der Verschuldungsgrenze wurde ein Vetorecht des im Jahre 2008 aufgestellten Budgetrates eingeführt. Der Budgetrat ist ein dreiköpfiges Verfassungsorgan, bestehend aus einem vom Präsidenten der Republik für sechs Jahre ernannten Präsidenten, dem Präsidenten der Ungarischen Nationalbank und dem Präsidenten des Staatsrechnungshofs (Art 43 Abs. 4 UGG). Dieser Rat prüft die Begründung des zentralen Staatshaushalts und die Einhaltung der Verschuldungsgrenze (Art. 43 Abs. 3 UGG). Ohne die Zustimmung dieses Rates kann das Budget nicht verabschiedet werden, was einem Vetorecht gleichkommt. Die Bedeutung dieses Vetorechts wird zusätzlich dadurch betont, dass der Präsident der Republik laut Art. 3 Abs. 3 lit. b) UGG das Parlament bei gleichzeitiger Ansetzung von Wahlen auflösen kann, wenn das Parlament den Staathaushalt bis zum 31. März - aus welchem Grund auch immer - nicht beschließt.

Die skizzierte Machtfülle der drei Mitglieder des Budgetrates wurde auch von der Venedig-Kommission aus demokratiepolitischer Sicht kritisiert. ${ }^{38}$ Diese Kritik ist nur teilweise berechtigt. Es ist wahr, dass die Existenz des Budgetrates den Gesetzgeber bevormundet. Allerdings hat sich das ungarische Parlament in den vergangenen zwei Jahrzehnten nicht durch Vorsicht, Zurückhaltung und fachliche Kompetenz ausgezeichnet, also kaum Zeichen eines vernünftigen Verhaltens gezeigt, was eine gewisse Vormundschaft im Bereich der Fiskalpolitik als notwendig erscheinen lässt. Dieselbe Kritik könnte man im Übrigen auch dem Verfassungsgericht gegenüber vorbringen, ist seine Existenz doch nur durch ein Misstrauen gegenüber der Politik zu erklären. ${ }^{39}$ Problematisch ist aber, dass

38 Commission for Democracy Through Law (Venice Commission) Opinion on the New Constitution of Hungary, Adopted by the Venice Commission at its 87th Plenary Session (Venice, 17-18 June 2011) Nr. CDL-AD(2011)016, Rn. 129.

39 Haltern, U.: Verfassungsgerichtsbarkeit, Demokratie und Misstrauen - Das Bundesverfassungsgericht in einer Verfassungstheorie zwischen Populismus und Progressivismus, Berlin, 1998, aus der ungarischen Literatur siehe Vincze, A.: 32/A § Az Alkotmánybíróság, a.a.O., Rn. 41-42. 
nicht einmal die Grundlinien des Verfahrens, der Methodik und der Entscheidungsmaßstäbe des Budgetrates aus dem Grundgesetz ersichtlich werden. In dieser Hinsicht muss das Parlament in einem Gesetz die notwendigen Garantien ausbauen. Zudem werden die persönlichen und fachlichen Qualitäten der Ratsmitglieder entscheidend sein.

Das allgemein zu begrüßende Ziel der Schuldenbegrenzung bringt aber auch Nebenwirkungen mit sich, die mit den herkömmlichen Vorstellungen von Rechtstaatlichkeit kaum zu vereinbaren sind. Laut Art 37. Abs. 4 UGG kann das Verfassungsgericht - solange die Staatsverschuldung nicht auf die Hälfte des BIP reduziert wird - die Verfassungsmäßigkeit von Gesetzen über den Staatshaushalt, über dessen Vollzug, über die zentralen Steuern, Gebühren, Abgaben und Zölle sowie über die zentralen Bedingungen der örtlichen Steuern ausschließlich im Hinblick auf das Recht auf Leben und Menschenwürde, das Recht auf Schutz der persönlichen Daten, das Recht auf Gedanken-, Gewissens- und Glaubensfreiheit oder die mit der ungarischen Staatsangehörigkeit verbundenen Rechte überprüfen und derartige Gesetze ausschließlich wegen einer Verletzung dieser Rechte für nichtig erklären. Die klar verständliche Nachricht dieser Vorschrift lautet, dass Rechtsstaatlichkeit aus Sicht der derzeitigen Regierung nicht jeden Preis rechtfertigt.

\section{Schlussbemerkungen}

Nach zwanzig Jahren rechtstaatlicher Demokratie war klar, an welchen Stellen die Verfassung von 1989 Erfolg hatte und wo sie verbesserungswürdig war, mithin einer Reform bedurfte. Statt einer solchen Anpassung, die Ungarn eine endgültige und europäische Verfassung hätte geben können, instrumentalisierte die rechtsorientierte Regierungskoalition ihre verfassungsändernde Zwei-DrittelMehrheit dazu, ihre Macht durch eine unzeitgemäße, rückwärtsgewandte Verfassung zu zementieren. Statt die Staatsmacht zu begrenzen - eine der wichtigsten Funktionen von Verfassungen - wird durch eine Mythologisierung der Vergangenheit versucht, dem Volk eine falsche nationale Identität aufzudrängen. Nachdem Premierminister Orbán im März 2012 die Europäische Union mit der Sowjetunion verglich, antwortete Kommissionspräsident Barroso, dass Orbán die Demokratie nicht verstehe. 